\section{PSICOLOGIA IBEROAMERICANA}

\section{Psicología lberoamericana}

ISSN: 1405-0943

psicología.iberoamericana@uia.mx

Universidad Iberoamericana, Ciudad de

México

México

Palacios Delgado, Jorge Raúl; Bustos Aguayo, José Marcos; Mercado Doménech, Serafín Implicaciones de las estrategias de adaptación sobre la conducta proambiental en jóvenes

Psicología lberoamericana, vol. 21, núm. 1, enero-junio, 2013, pp. 65-78

Universidad Iberoamericana, Ciudad de México

Distrito Federal, México

Disponible en: http://www.redalyc.org/articulo.oa?id=133929862008

Cómo citar el artículo

Número completo

- Más información del artículo

- Página de la revista en redalyc.org

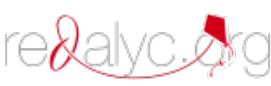

Sistema de Información Científica

Red de Revistas Científicas de América Latina, el Caribe, España y Portugal Proyecto académico sin fines de lucro, desarrollado bajo la iniciativa de acceso abierto 


\title{
Implicaciones de las estrategias de adaptación sobre la conducta proambiental en jóvenes ${ }^{1}$
}

\author{
Implications of Environment Adaption on Proenvironmental \\ Behavior in Youths
}

\author{
Jorge Raúl Palacios Delgado* \\ José Marcos Bustos Aguayo \\ Facultad de Estudios Superiores Zaragoza \\ Serafín Mercado Doménech \\ Universidad Nacional Autónoma de México
}

\section{RESUMEN}

Los objetivos de la presente investigación fueron identificar, mediante el análisis del escalamiento dimensional, los nichos ecológicos presentes en el ecosistema humano y determinar si las características de adaptación influyen en la conservación proambiental y en el consumo ambientalmente responsable en una muestra de jóvenes. Se evaluó la adaptación ambiental con una escala diseñada para este estudio, además se midió la conducta proambiental y el consumo ambientalmente responsable con escalas validadas en población mexicana. Se seleccionó una muestra de 700 jóvenes de entre 13 y 24 años de edad (media= 16.48) de la zona metropolitana de la Ciudad de México. El escalamiento multidimensional confirmó cuatro dimensiones claramente interpretables: adaptación al entorno natural, sociocultural, urbano e individual. La regresión múltiple evidenció que los jóvenes que se adaptan al medio natural y sociocultural realizan acciones para conservar el ambiente, además quienes se adaptan a la naturaleza y se ajustan al entorno urbano realizan compras amigables con el ambiente. Se discute la utilidad de los resultados a partir de la conservación ambiental en jóvenes, así como las implicaciones para futuros estudios.

Palabras clave: adaptación ambiental, conducta proambiental, consumo responsable, escalamiento multidimensional y jóvenes.

\section{ABSTRACT}

The objective of this research was to identify by means of multidimensional scaling analysis the ecological niches into the human ecosystem and establish how the adaptation characteristics predict the proenvironmental behavior and environmental sustainable consumption in youths. The environmental adaptation was measured in a newly development scale for the purpose of the present research. The proenvironmental scale $(\alpha=.82)$ and environmental sustainable consumption $(\alpha=.78)$ were also included in the study. The sample was constituted by 700 youths between 13 and 24 years old (mean 16.48), in México City and Metropolitan area. The results confirmed by the multidimensional scaling four interpretable factors (natural adaption, sociocultural adaption, urban adaption and personal adaption). The regression analyses suggested the youths that use natural and sociocultural adaptations realize proenvironmental actions as well as the youths there was adapted a natural and urban context make different types of environmental sustainable consumption. The utility of the results are discussed from the evolutionary perspective and the implications for the adolescents are suggested.

Keywords: environmental adaptation, proenvironmental behavior, environmental sustainable consumption, multidimensional scaling and youths.

\footnotetext{
${ }^{1}$ La presente investigación forma parte del proyecto Modelo Biopsicosocial del Comportamiento Proambiental, financiado por el programa de estancias posdoctorales de la Dirección General de Asuntos del Personal Académico (DGAPA), Universidad Nacional Autónoma de México. El segundo autor agradece a la DGAPA el apoyo económico otorgado para realizar el proyecto PAPIIT IN308811.

"Recibido: 5 de octubre, 2012 - Aceptado: 22 de marzo, 2013.

Correspondencia: Dr. Jorge Raúl Palacios Delgado, Facultad de Estudios Superiores Zaragoza, unAM; Guelatao 66, col. Ejército de Oriente, Delegación Iztapalapa, C. P. 09230.

e-mail: kobuj@yahoo.com.mx, teléfono: 57456821.
} 


\section{INTRODUCCIÓN}

La psicología evolutiva es un nuevo paradigma que incorpora los principios modernos de la psicología con los principios evolutivos de la biología (Buss, 1995; 2009). Los avances teóricos y metodológicos recientes de este paradigma muestran un desarrollo prominente en temas como la elección de pareja (Buss \& Barnes, 1986), la personalidad (Buss, 1991), la sexualidad (Buss, 1995), la agresión (Buss \& Shackelford, 1997), las diferencias individuales (Buss \& Greiling, 1999), el apego romántico (Schmitt, 2008), la supervivencia (Buss, 2009), el altruismo (Tal, Hill, Figueredo, Frías \& Corral, 2006), el manejo del humor (Li, Griskevicius, Durante, Jonason, Pasiszand \& Aumer, 2009), y las emociones positivas (Griskevicius, Shiota \& Nowlis, 2010).

Para la psicología evolutiva una de las funciones del individuo es la adaptación al entorno que le rodea. En sociología y psicología, la adaptación es el proceso por el cual un grupo o un individuo modifican sus patrones de comportamiento para ajustarse a las normas establecidas en el medio social en el que se encuentran (Martín, 1993). Para Granada (2003), la adaptación cultural implica el proceso de incorporación de un individuo a las normas de conducta del grupo al que pertenece; incluye también el proceso de recepción de otra cultura (aculturación) y de adaptación al nuevo contexto sociocultural o sociolingüístico.

La psicología evolutiva (Buss, 2009; Griskevicius, Cantú \& van Vugt, 2012) también provee una nueva conceptuación de problemas adaptativos (situaciones) que han sido confrontados y solventados por los seres humanos, utilizando mecanismos psicológicos especialmente sensitivos a claves que los preparan para desplegar estrategias conductuales que les sirven para resolver problemas. De esta manera, la evolución ha dotado de características a los individuos, que van desde variaciones genéticas (Tobby \& Cosmides, 1990) y rasgos de personalidad (Buss, 1996; MacDonald, 1995), hasta estrategias de afrontamiento (Lazarus \& Folkman, 1986) para resolver problemas adaptativos. Desde el punto de vista evolutivo (Buss, 1984; 1995; 1996; Tobby et al., 1990), el repertorio conductual de las personas emerge de una constante interacción entre los problemas encontrados en los ecosistemas y el funcionamiento de los organismos para sobrevivir y reproducirse en los nichos ecológicos que habitan.
Algunos nichos requieren que ciertos elementos del ambiente cambien para que su funcionamiento pueda ser el óptimo (Buss, 1984; Buss, 2009).

De acuerdo con Díaz-Loving (1998; 2008; 2011), a través de la evolución la composición genética y los rasgos de personalidad de los seres humanos, han sido moldeados por ecosistemas específicos, así como por las normas o valores derivados de características idiosincráticas del grupo social. Además, la evolución humana se encuentra inmersa en procesos de socialización y endoculturación que han dado por resultado una serie de similitudes o diferencias que se encuentran en nichos ecológicos particulares, herencias culturales, predisposiciones biológicas y experiencias personales que interactúan entre sí para producir el comportamiento social. Según lo explican Mercado, Landázuri y Terán (2006), el humano transforma activamente el entorno y lo adapta a sus necesidades o requerimientos, ocupando una amplia variedad de hábitats.

Para Díaz-Guerrero (1988; 2001), las características del individuo se explican a partir del ecosistema cultural, postulando que la sociedad y las comunidades humanas son un complejo sistema de interdependencia análogo al ecosistema natural en biología. Las características psicológicas de los individuos derivadas del ecosistema cultural incluyen, entre otras, el estilo de confrontación individual frente a los problemas que plantea el ecosistema (filosofía de vida) y el grado de acuerdo con las premisas propias de la cultura (DíazGuerrero, 1995). Las normas y principios socioculturales de los grupos de referencia y afiliación (familia, grupo, sociedad) son una guía de comportamiento (Díaz-Guerrero, 1994), de esta manera, dentro de estas premisas culturales, existen dos estilos de confrontación o maneras de encarar los problemas de la vida, ya sea de forma activa o bien de forma pasiva (Díaz-Guerrero, 1984); la forma pasiva de confrontación parece ser un factor cardinal en la personalidad de los mexicanos (Díaz-Guerrero, 1993), comparada con culturas individualistas (Díaz-Guerrero, 1994; Triandis, 1996).

Por otra parte, la psicología ambiental estudia la relación del ambiente físico, natural y construido, con la conducta humana (Mercado et al., 2006). Considera todo aquello que rodea a las personas, las interacciones entre los individuos y sus escenarios físicos (Gifford, 2007), con particular interés en la relación del hombre 
con la naturaleza y con el espacio construido. Busca conocer de qué manera las situaciones ambientales afectan la conducta, el desarrollo de las personas y cómo la conducta de estos transforma o altera el medio (Corraliza y Berenguer, 2010; Schmuck \& Vlek, 2003). También investiga la adaptación de los organismos al entorno y trata de resolver los problemas de adaptación del hombre en relación con el entorno sociofísico. Además, pretende deducir el problema de los sujetos a los escenarios conductuales y a las normas sociales de la cultura (Mercado et al., 2006).

La psicología ambiental resulta elemental para explicar los problemas ecológicos y la forma que tienen los organismos para incorporarse al entorno a través de su capacidad para integrar información, no sólo del ambiente natural, sino del entorno diseñado o construido (Schmuck et al., 2003) y se centra en el análisis de las maneras en que el medio ambiente afecta al individuo y el modo en que las acciones de los sujetos transforman el entorno o la relación con el mismo (Oskam, 2000). Bechtel (1997) indicó que las alteraciones ambientales y daños a los ecosistemas requieren formas de respuesta humana que favorezcan la sobrevivencia de la especie, lo cual es congruente con una perspectiva que considera las bases evolutivas para la conducta sustentable (Griskevicius et al., 2012). En este sentido, algunos autores (Griskevicius et al., 2012; Griskevicius, Tybur \& Van den Bergh, 2010) consideran que la sustentabilidad y su contraparte, el daño al medio ambiente, pueden ser explicados por medio de la conducta del individuo, así como por estrategias evolutivas que utiliza el mismo; de esta manera Griskevicius et al. (2010), explican porque las personas compran productos ecológicos frente a productos convencionales, argumentando que desde el punto de vista biológico, el altruismo se asocia a una señal de estatus, y éste motiva el deseo de comprar productos ecológicos cuando se realiza en lugares públicos (pero no en privado). Estos productos cuestan más que los productos no ecológicos. Concluyen señalando que el estatus se vincula con la competencia y puede ser usado para promover conducta proambiental.

Adicionalmente a lo anterior, Brown (2009) señaló la necesidad de realizar cambios en el patrón de consumo para evitar mayores daños a los ecosistemas y con ello, evitar los riegos que confrontaran las nuevas gene- raciones. Los cambios que se requieren son la sustitución de energía fósil por renovable, la reducción de la contaminación del agua de ríos y mares, la producción eficiente de alimentos, la protección de la biodiversidad, y la disminución de la desigualdad social, en tanto, los grupos y los individuos tendrán que ajustar sus estilos de vida realizando conductas proambientales, ahorrando agua y energía eléctrica, reciclando los residuos, viajando en transporte sustentable, entre otras (Bustos, 2004; Bustos \& Flores, 2006; Bustos, Flores \& Barrientos, 2008; Palacios \& Bustos, 2011; Palacios \& Bustos, 2012; Palacios \& Bustos, en prensa).

Los espacios creados por el hombre, ya sean viviendas, unifamiliares, condominios, fábricas, hospitales, cárceles, escuelas, etc., contienen un sistema de significados explícitos o implícitos que informan a los usuarios acerca del sistema de valores, creencias, actitudes e intenciones de los habitantes (Corraliza \& Berenguer, 2010). El conocimiento de estos elementos resulta indispensable desde el punto de vista adaptivo para que su habitabilidad sea idónea para el bienestar del hombre y su mejor calidad de vida (Corral, 2010; Schmuck et al., 2003). Los sitios son diseñados o equipados para que los encuentros sociales se lleven a cabo, con una correspondencia entre la distribución arquitectónica o urbanística, el mobiliario y las conductas que se esperan tengan lugar allí. Estas conductas forman parte del entorno social, definido como la parte del ambiente de un individuo constituida por otros seres humanos y la red de relaciones que los vincula.

Paralelamente, uno de los problemas del entorno social es la elevada densidad urbana, que ha tenido como resultado la pérdida de espacios verdes, que sirven como nodos de contacto con la naturaleza (Home, Bauer \& Hunziker, 2010). Los espacios construidos han ganado terreno a las áreas verdes, lo que ha provocado la reducción de escenarios necesarios para la restauración cognitiva y emocional de los residentes (Hartig, 2011; Martínez \& Montero, 2010). En consecuencia, se hace indispensable mantener estos espacios públicos y generar en lo posible otros nuevos (Flores \& GonzálezGuillén, 2007; Landázuri, Lee, Terán \& Mercado, 2010), dada su importancia para la salud física y mental. La adaptación supone proteger los elementos naturales y las áreas verdes que benefician a los residentes, además de ajustarse a los cambios exigidos por medio natural. 
Una forma esencial de responder a esta exigencia es promoviendo las acciones de protección del entorno tanto construido como natural, donde se entremezclan los espacios protegidos como las reservas ecológicas o los espacios públicos, como los parques urbanos (Bustos, Ramos, Barrón, López \& Ocaña, 2011).

Los individuos poseen diferentes formas para integrarse al entorno que los rodea a través de las estrategias de adaptación (Buss, 1991; 1996). La adaptación a un contexto es un proceso que requiere un cambio en el comportamiento para poder habituarse al nuevo ambiente. Los ambientes (nichos) que existen pueden ser creados específicamente para desarrollar los atributos de los organismos (Buss, 1984). De esta manera, la conducta de los individuos se explica por las situaciones en las que se encuentra, las cuales implican lugares y tiempos determinados donde se llevan a cabo situaciones sociales programadas por la cultura (Mercado et al., 2006; Díaz-Guerrero, 1994).

Derivado de lo que se ha expuesto a lo largo de este artículo, se proponen tres vertientes de investigación vinculadas con la adaptación: la relación del hombre con el entorno natural, el ambiente que el hombre diseña y construye para resguardar su vida social, y por último, las relaciones que el individuo tiene en su entorno sociocultural para ajustar su conducta al entorno en el que se encuentra.

Los tres ejes de análisis mostrados hasta el momento llevan a plantearnos las siguientes interrogantes: ¿existe una serie de nichos ecológicos preexistentes a los cuales los individuos se han enfrentado para su supervivencia?, ¿cuáles son las estrategias de adaptación que el hombre ha desarrollado para integrarse a ecosistemas naturales, diseñados o construidos?, ¿qué relación mantiene el ser humano con sus semejantes en estos entornos?, ¿qué características de adaptación tienen los jóvenes en México que les permiten realizar acciones de conservación ambiental?

Con la finalidad de dar respuesta a las interrogantes anteriores y con el propósito de conocer los entornos (nicho ecológico) a los cuales las personas se pueden adaptar, los objetivos de la presente investigación fueron identificar, mediante escalamiento dimensional, los nichos ecológicos que se encuentran presentes en el ecosistema humano, describir las características de adaptación de los jóvenes en su entorno natural o construido, así como determinar si las características de adaptación influyen en la conservación proambiental y el consumo ambientalmente responsable en una muestra de jóvenes. Con base en la hipótesis nula, se plantea que las características de adaptación de los jóvenes no tienen un efecto en acciones de conservación proambiental (CPA) y en el consumo ambientalmente responsable.

\section{MÉTODO}

\section{Participantes}

En el estudio participaron 700 jóvenes, 291 hombres y 409 mujeres, con un rango de edad entre los 13 y los 24 años $(M=16.48$ años; $\mathrm{DE}=2.7$ años), seleccionados mediante un muestreo no probabilístico de tipo accidental. Los jóvenes eran estudiantes de varias escuelas de educación media, media superior y superior, tanto públicas como privadas, de la zona metropolitana de la Ciudad de México, de ambos turnos, el 75.9\% del turno matutino y $24.1 \%$ del vespertino.

\section{Instrumento}

Para evaluar la adaptación ambiental se redactaron 21 afirmaciones tipo Likert con cuatro opciones de respuesta (nunca a siempre) sobre diferentes entornos a los cuales los individuos se pueden adaptar. Se consideraron los ecosistemas naturales o biológicos (p. e. Para formar parte de la naturaleza me adapto a ella), los citadinos (p. e. Me acostumbro con facilidad al tráfico de la ciudad) y los socio-culturales (p. e. Frente los problemas lo mejor es acomodarse a ellos). Los ítems se redactaron considerando que su contenido fuera congruente con la propuesta de los nichos naturales (Mayer y Frantz, 2004; Palacios y Bustos, 2012), urbanos (Mercado et al., 2006; Home et al., 2010) y socioculturales (DíazGuerrero, 1984, 1988, 1995) planteados en el presente estudio y que pudieran reflejar repertorios de adaptación de los jóvenes. Para esta investigación definimos la adaptación ambiental como las características que un individuo posee para acomodarse o ajustarse a diversas circunstancias o condiciones de su entorno (nicho ecológico) a través del proceso evolutivo (selección natural) y cultural.

Para medir la conducta proambiental (CPA) se utilizó la escala de conducta proecológica de Corral, 
Tapia, Frías, Fraijo y González (2009). Es una escala tipo Likert, con 12 reactivos en la que los participantes reportan la frecuencia de comportamientos de cuidado del ambiente (p.e. guardo y reciclo papel usado). Se contesta con cuatro opciones de respuesta que van de nunca hasta siempre. Cuenta con validez factorial y predictiva, así como con un coeficiente de confiabilidad por consistencia interna de .82 para el total del instrumento.

La medición del consumo ambientalmente responsable se realizó con la escala de Bustos, Palacios, Barrientos y Flores (en prensa). Es una escala tipo Likert con cuatro opciones de respuesta (nunca a siempre) que mide tres factores del consumo ambiental. La escala tiene validez de constructo y un coeficiente de confiabilidad por consistencia interna (alfa de Cronbach) para el total del instrumento de .86 (IC95\%=.84-.88). La escala original consta de un total de 15 ítems y para el presente estudio únicamente se utilizaron tres afirmaciones (p.e. Adquiero productos que son más amigables con el ambiente) con un $\alpha=.78$.

\section{Procedimiento}

Se aplicó el cuestionario a los jóvenes de manera grupal, utilizando a los grupos escolares para tal fin. Se les pidió que respondieran a un cuestionario elaborado para conocer algunas actividades relacionadas con el medio ambiente que realizan las personas de su edad. Se les aclaró que su participación era voluntaria, que no había respuestas buenas ni malas y que la información era anónima, para lo cual se les solicitó que contestaran de forma sincera, explicándoles que sus respuestas se utilizarían para fines de investigación. Asimismo, se resolvieron las dudas que tuvieran. La duración total para responder el instrumento fue de 20 minutos, aproximadamente.

\section{RESULTADOS}

Para evaluar las características psicométricas del instrumento de adaptación ambiental, en primer lugar se revisó la distribución de frecuencias de las respuestas de cada ítem para conocer si los ítems discriminaban, estaban sesgados o tenían una distribución asimétrica. En segundo lugar, se seleccionaron los ítems que tuvieran la correlación ítem total más alta o superior a .40 para considerarlos como indicadores de la esca- la de adaptación ambiental. De este procedimiento se seleccionaron todos los ítems, ya que cumplieron con los criterios establecidos. Posteriormente se realizó un escalamiento multidimensional para identificar las dimensiones subyacentes entre las estrategias que se encuentran dentro de la escala de adaptación.

Se optó por esta técnica multivariante para poder inferir dimensiones o similitudes realizadas por los jóvenes sobre las estrategias de adaptación que poseen. Además, permitió a la investigación: 1) determinar qué posibles dimensiones utilizan los encuestados, 2) observar cuántas dimensiones pueden utilizarse en una situación particular, 3) conocer la importancia relativa de cada dimensión, 4) indagar cómo se relaciona perceptualmente cada estrategia y 5) en caso necesario, permite obtener una solución para cada individuo (Hair, Anderson, Tatham \& Black, 1999).

A pesar de que la técnica no utiliza un valor teórico per se, la evidencia mostrada indica al menos tres ejes para su posible análisis: la relación del hombre con el entorno natural y con el entorno construido, así como las relaciones que el individuo lleva a cabo en su entorno sociocultural.

Para obtener las dimensiones de la adaptación ambiental se utilizó el método (algoritmo) ALSCAL (Arce, Francisco \& Arce, 2010). Ell número de dimensiones se estableció considerando el cambio de los valores de s-stress y del índice de correlación al cuadrado $(R S Q)$. En la tabla 1 se detallan dos índices de bondad de ajuste obtenidos para las diferentes soluciones. El análisis de escalamiento multidimensional permitió identificar de dos a cuatro dimensiones. Al pasar de dos a cuatro dimensiones, la mejoría del $s$-stress sugiere que la inclusión de la cuarta dimensión produce el mayor decremento del stress y el mayor incremento de la varianza explicada ( $R S Q)$. De esta manera, la solución más eficiente es la de cuatro dimensiones.

Con el fin de poder visualizar mejor los resultados obtenidos, la figura 1 muestra el gráfico de la solución multidimensional (se optó por colocar la gráfica bidimensional, ya que sirvió de base para la interpretación de las dimensiones). La interpretación de las dimensiones se realizó considerando en primer lugar el centroide (los ítems que ocupan la posición más central dentro del gráfico; en este caso el ítem adapta 55) y la semejanza con los ítems cercanos. Posteriormente se 
Tabla 1. Niveles de ajuste para las distintas soluciones dimensionales

\begin{tabular}{c|c|c|c}
\hline Índices de ajuste & Dos dimensiones & Tres dimensiones & Cuatro dimensiones \\
\hline S-stress & .17091 & .10831 & .07435 \\
\hline RSQ & .85094 & .92011 & .95324 \\
\hline
\end{tabular}

consideraron los conceptos que ocupan las posiciones mas extremas del centroide y de cada dimensión. De esta manera se puede observar en la dimensión 1 (eje vertical) que las características centrales de la adaptación, es decir, los más representativos del constructo por estar mejor correlacionados con todos los demás, corresponden de forma predominante a la adaptación del contexto en el que se encuentran los jóvenes, incorporando acciones señaladas por su familia, amigos o vecinos (adapta 38), acostumbrándose a estar en lugares donde hay muchas personas juntas (adapta 40), acomodándose a los problemas cuando se les presentan (adapta 54) y ajustando su forma de ser si no va de acuerdo a la situación (adapta 51). A la derecha del centroide se encuentran situadas las características que permiten a los individuos adaptarse a los problemas de la vida diaria (adapta 52), acomodarse al medio que les rodea (adapta 53), a las condiciones del ambiente (adapta 39) y ajustarse a los cambios en las actividades de su vida cotidiana (adapta 57).

$\mathrm{Al}$ interpretar la dimensión 2 (eje horizontal) se encuentra que ésta se subdivide en dos partes; la primera del lado izquierdo ubica un conglomerado representado por el ítem 48, que es el más alejado del centroide que hace referencia a características de adaptación vinculadas a la forma en la que los individuos se acostumbran con facilidad al tráfico de la ciudad, a vivir con la contaminación del aire y/o de las calles (adapta 49), así como al ruido de autos y camiones con facilidad (adapta 47). Además, cuando los jóvenes se acostumbran a estas características del entorno urbano, cambian su manera de ser para formar parte del entorno medioambiental (adapta 41), como estrategia para adaptarse a este nuevo medioambiente que les de-

Figura 1. Escalamiento multidimensional de la adaptación ambiental

\section{Configuración de estímulos derivada}

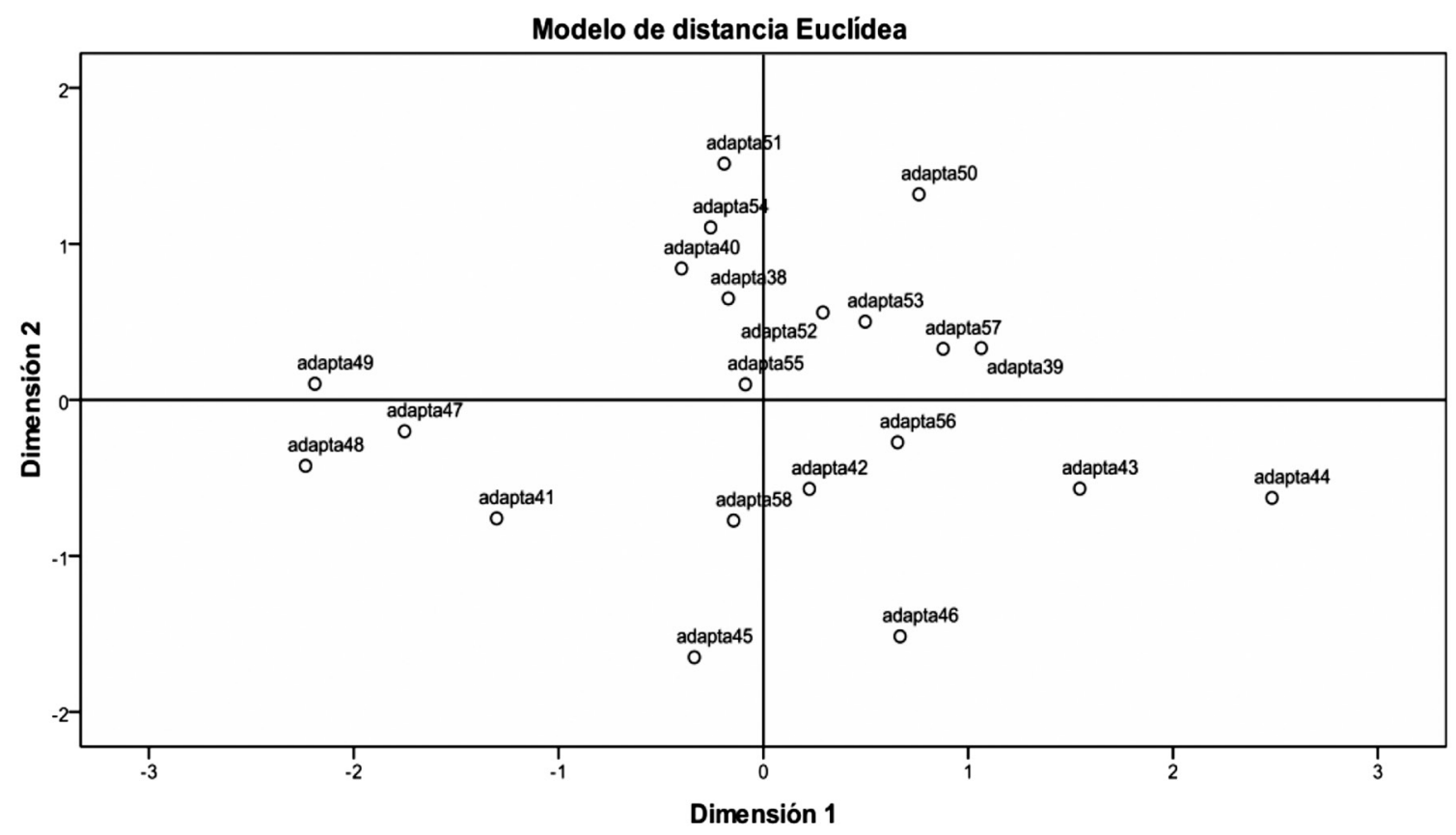


manda ajustarse a vivir con poca agua y sobrevivir a ello (adapta 45). En el polo opuesto de la dimensión 2 , existe un cúmulo de características que en primera instancia se relacionan con el conglomerado del lado izquierdo, como es la utilización de poca luz eléctrica (adapta 46) y acostumbrarse a los cambios de clima fácilmente (adapta 42). Después de éstas, se ubican características adaptativas definidas por el ajuste a formar parte de la naturaleza (adapta 56), adaptarse a convivir con ésta (adapta 43), así como a vivir en lugares con vegetación y llenos de naturaleza (adapta 44).

La configuración obtenida mediante el escalamiento permitió establecer cuatro dimensiones, la primera fue nombrada adaptación sociocultural (5 ítems, $\alpha=.67$; IC95\%= $0.63-0.71$ ), la segunda corresponde a la adaptación individual-social (5 ítems, $\alpha=.72$; IC95\% $=0.69-0.75)$, la siguiente se refiere a la adaptación al entorno urbano o construido ( 6 ítems, $\alpha=.70$; IC95\% $=0.66-0.73)$ y la última representa la adaptación al entorno natural ( 5 ítems, $\alpha=.73$; IC95\% $=0.70$ 0.76). Estas dos últimas dimensiones parecen formar un continuo de adaptación que va de lo urbano a lo natural o viceversa. Para conocer el nivel de confiabilidad por consistencia interna, se calculó el coeficiente alfa de Cronbach para el total del instrumento, obteniendo un índice de 0.87 (IC95\%= 0.86- 0.88).

Se realizaron correlaciones multivariadas producto momento de Pearson (tabla 2), con lo que se encontraron relaciones positivas y significativas entre las dimensiones de la adaptación, mostrando que los jóvenes que se adaptan al contexto sociocultural, incluido lo señalado por su familia o amigos, y que enfrentan los problemas que se les presentan cotidianamente, se ajustan a su medio social utilizando estrategias de adaptación de las adversidades en sus actividades diarias. Por medio de características personales, tienden a adaptarse en mayor medida al entorno urbano que incluye el tráfico de la ciudad, seguido del entorno natural que incorpora vivir en lugares con vegetación y llenos de naturaleza. Los jóvenes que se adaptan a los problemas de la vida diaria o se acomodan al medio que les rodea, se acostumbran a convivir con la naturaleza y al ruido de autos y camiones fácilmente. Por último, los jóvenes que se ajustan a convivir con la naturaleza o que se acostumbran a utilizar poca luz eléctrica, cambian su repertorio comportamental para adaptarse al entorno urbano, que incluye la contaminación de las calles y el tráfico de la ciudad.

Adicionalmente, se encontraron relaciones moderadas y positivas entre el consumo ambiental responsable, la conducta proambiental y las cuatro estrategias de adaptación, es decir, los jóvenes que se preocupan por cuidar el ambiente cuando realizan compras y son responsables con éste, llevando a cabo acciones para su conservación, despliegan repertorios conductuales de adaptación al estar acostumbrados a realizar acciones que les indican su familia, amigos o vecinos; se ajustan a los cambios en las actividades de su vida cotidiana,

Tabla 2. Estadística descriptiva y correlaciones de la escala de adaptación ambiental

\begin{tabular}{l|c|c|c|c|c|c|c|c}
\cline { 2 - 8 } & $\mathbf{1}$ & $\mathbf{2}$ & $\mathbf{3}$ & $\mathbf{4}$ & $\mathbf{5}$ & $\mathbf{6}$ & Media & Desviación estándar \\
\hline Adaptación sociocultural & -- & $.67^{*}$ & $.52^{*}$ & $.40^{*}$ & $.25^{*}$ & $.28^{*}$ & 12.32 & 3.0 \\
\hline Adaptación individual-social & & -- & $.48^{*}$ & $.52^{*}$ & $.27^{*}$ & $.30^{*}$ & 13.49 & 2.9 \\
\hline Adaptación urbana & & & -- & $.49 *$ & $.32^{*}$ & $.32 *$ & 13.05 & 3.5 \\
\hline Adaptación natural & & & & -- & $.47^{*}$ & $.52^{*}$ & 12.90 & 3.3 \\
\hline Consumo ambiental & & & & & -- & $.56^{*}$ & 6.92 & 2.2 \\
\hline Conducta proambiental & & & & & & -- & 28.54 & 6.7 \\
\hline
\end{tabular}

${ }^{*} p<.001$ 
incluido el tráfico de la ciudad y los cambios de clima fácilmente, además de esto, son capaces de adaptarse a convivir con la naturaleza.

Las estrategias de adaptación sirvieron de criterios para predecir la conducta proambiental y el consumo ambientalmente responsable de los jóvenes mediante un análisis de regresión múltiple de pasos sucesivos. En las tablas 3 y 4 se muestran los principales resultados en las dos regresiones paso por paso. Los dos análisis de regresión utilizaron como predictores las cuatro dimensiones de la adaptación ambiental y se consideraron como criterios en primer lugar, la conducta proambiental y en segundo, la ambientalmente responsable.

Para la conducta proambiental se identificaron dos predictores independientes y significativos que entraron en el modelo de regresión,; en el primer paso entró la adaptación natural ( $F=209.40, p<.001)$ como el mejor predictor. En el segundo, se incorporó la adaptación sociocultural en la ecuación de regresión ( $F=107.47, p<.001)$. Lo anterior significa que los jóvenes que se adaptan a la naturaleza y al contexto sociocultural realizan más acciones de conservación ambiental. Ambas características de adaptación explican un $23.6 \%$ de la varianza de la conducta proambiental.

Tabla 3. Análisis de regresión múltiple paso por paso para predecir la conducta proambiental

\begin{tabular}{|c|c|c|c|}
\hline Variable & B & SE B & $\beta$ \\
\hline \multicolumn{4}{|l|}{ Paso 1} \\
\hline Adaptación natural & .88 & .07 & $.44 * *$ \\
\hline \multicolumn{4}{|l|}{ Paso 2} \\
\hline Adaptación sociocultural & .16 & .07 & $.07^{*}$ \\
\hline
\end{tabular}

El modelo de regresión de la adaptación ambiental sobre el consumo responsable con el ambiente mostró dos predictores independientes y significativos. En el primer paso entró la adaptación natural ( $F=194.39$, $p<.001)$ como el mejor predictor. En el segundo, se incorporó la adaptación urbana en la ecuación de regresión $(F=403.13, p<.001)$, es decir, aquellos que se adaptan a la naturaleza y se ajustan al entorno urbano realizan compras amigables con el ambiente. Estas características de adaptación explican un $23.1 \%$ de la varianza del consumo ambiental responsable.

Tabla 4. Análisis de regresión múltiple paso por paso para predecir el consumo ambientalmente responsable

\begin{tabular}{|c|c|c|c|}
\hline Variable & B & SE B & $\beta$ \\
\hline \multicolumn{4}{|l|}{ Paso 1} \\
\hline Adaptación natural & .27 & .02 & $.40 * *$ \\
\hline \multicolumn{4}{|l|}{ Paso 2} \\
\hline Adaptación urbana & .08 & .02 & $.13^{*}$ \\
\hline
\end{tabular}

${ }^{*} p<.05 *{ }^{*} p<.01$

\section{DISCUSIÓN}

Desde el punto de vista evolutivo (Buss, 1984, 1995, 1996; Tobby et al., 1990), la adaptación involucra soluciones a los problemas de supervivencia y reproducción; debido a ello, los individuos han desplegado distintas estrategias y repertorios conductuales para adaptarse a los diferentes nichos o ecosistemas que involucran escenarios de conducta y sociales que, con el paso del tiempo, se han relacionado con aspectos tanto socio-culturales como individuales y naturales, dando lugar a una interdependencia entre el espacio natural o diseñado, la conducta de los sujetos y las relaciones entre ellos.

Mediante el escalamiento multidimensional se exploró una configuración de dimensiones que permitió averiguar el significado conceptual y de constructo de la adaptación a diferentes ecosistemas, a los cuales los individuos se pueden ajustar. Con base en la técnica de escalamiento dimensional se obtuvieron cuatro áreas de adaptación en esta muestra de jóvenes mexicanos. Las dimensiones encontradas fueron nombradas: adaptación sociocultural, adaptación individualsocial, adaptación urbana y adaptación a lo natural, y mostraron consistencia interna con coeficientes de confiabilidad alfa de Cronbach entre .67 y .73 . Aunque el índice de confiabilidad se encuentra al límite de lo aceptable, lo cual se atribuye posiblemente a los pocos ítems contenidos en cada dimensión, parece conveniente en un futuro mejorar el nivel de consistencia interna, incorporando nuevas afirmaciones que muestren la variabilidad de los constructos encontrados en las escala de adaptación ambiental. 
Las dimensiones encontradas permiten dar validez de constructo al instrumento elaborado para evaluar las estrategias de adaptación ambiental, ya que estas se caracterizaron por mostrar cierta congruencia conceptual. Las correlaciones interescala complementan la validez de contenido derivada de diferentes posturas (Díaz-Guerrero, 1984; 1988; 1995; Home et al., 2010; Mayer et al., 2004; Mercado et al., 2006; Palacios et al., 2012).

Además, cada una de las dimensiones retenidas ofrece evidencia acerca de las diferentes estrategias de adaptación que los adolescentes utilizan para ajustarse a su entorno. La configuración multidimensional obtenida en la medición de la adaptación ambiental permite dar respuesta a las primeras interrogantes planteadas al inicio del estudio, sobre las estrategias de adaptación que se han desarrollado para integrarse a ecosistemas naturales, diseñados o construidos, y la relación que mantiene el ser humano con sus semejantes en estos entornos. Los resultados permiten señalar que los jóvenes se integran a distintos nichos que se les presentan, como son: el ecosistema natural (Buss, 1984; Bustos, 2011; Díaz-Guerrero, 1988; Palacios et al., 2012), la protección del mismo (Bechtel, 1997; Bustos, 2004; Bustos et al., 2006; Palacios et al., 2011; Schmuck et al., 2003), el ecosistema individual y sociocultural (DíazGuerrero, 1988; 1993; 1995; 2001; Díaz-Loving, 1998; 2008; 2011; Granada, 2003), en donde se mezclan y se ponen a prueba ciertas demandas para que el individuo se acomode o ajuste su comportamiento, dependiendo del ecosistema al cual tiene que adaptarse, ya que por un lado, responde como ser biológico con necesidades definidas evolutivamente, y por el otro, da respuesta a las demandas socioculturales que exigen un cambio en los estilos de vida, los cuales deben ser ajustados a vivir de modo sustentable.

Lo anterior se deriva de que los ecosistemas son redes de interacción entre los organismos (factores bióticos) y su entorno físico (factores abióticos) (DíazGuerrero, 1995; Mercado et al., 2006). Los aspectos del entorno (natural y diseñado) intervienen en los procesos de relación de cada ser humano con sus semejantes y consigo mismo (Díaz-Guerrero, 2001; Palacios et al., 2012). Desde el punto de vista psicológico, hay que considerar las variables intermediarias entre el ambiente y la reacción de los individuos en las situaciones en las que se encuentran (Mercado et al., 2006), ya que, dependiendo del grado en que un evento se percibe como amenazante, dañino o desafiante (Lazarus et al., 1986), interviene un conjunto de variables mediadoras en la evaluación de la anticipación de sus consecuencias y la evaluación de sus costos.

Es a través de las características de adaptación que las personas responden a exigencias de diversa índole, $y$ en el caso que se reporta en el presente estudio, se observa que éstas provienen del medio en sus nichos social, natural y urbano. Biológicamente, el humano responde a los cambios en su entorno, ya sea que éstos representen transformaciones atmosféricas, amenazas al bienestar físico, y riesgos de afectación por deslaves, sequías, terremotos, inundaciones, u otras amenazas; al mismo tiempo que puede verse afectado por las amenazas de tipo tecnológico, como la contaminación atmosférica o el ruido de las máquinas.

La adaptación al ambiente natural-construido requiere enfrentar demandas que la sociedad ha resuelto en cierta medida, al responder a las amenazas naturales, enfermedades, inclemencias climáticas, etc., que el propio hombre ha ayudado a crear desarrollando nuevas amenazas y riesgos para sí. Como se habrá observado, la adaptación también es personal y social, con lo que se habrán de reforzar culturalmente valores de sustentabilidad, bajo los cuales, de forma paralela, los jóvenes se tendrán que adaptar y ajustarse a las modificaciones ambientales de la actualidad, como las alteraciones en el clima, la contaminación atmosférica, la contaminación de los ríos, mares y lagos. Así que, la protección del medio ambiente y de la naturaleza, así como una "vida austera" serán las mejores formas de adaptarse a los efectos actuales del cambio climático para buscar modos de vida amigables con el ambiente. La forma de respuesta social, desde una perspectiva de sustentabilidad (Corral, 2010), que se ha enfatizado ante los cambios provocados por el deterioro del entorno natural, es el desarrollo de la conducta proambiental en sus diversas clases (Bustos et al., 2006; Bustos et al., 2008; Palacios et al., 2012; Palacios et al., en prensa). Las estrategias de austeridad, uso y consumo responsable de los recursos naturales, se requieren en mayor medida, no sólo en el ámbito doméstico y laboral, sino en diversas áreas de las actividades humanas (Schmuck et al., 2003). 
Por otro lado, la validez referida a un criterio o predictiva de la evaluación de la escala de adaptación ambiental propuesta en este estudio, permitió determinar la influencia que la adaptación tiene sobre la CPA y el consumo responsable con el ambiente. Los datos encontrados sugieren que la adaptación a los nichos naturales y socioculturales tienen un efecto directo sobre la conducta proambiental, es decir, los jóvenes que, por una parte, utilizan poca luz eléctrica en su vida diaria, que se han acostumbrado a los cambios de clima como forma de convivir con la naturaleza y que por otra parte, incorporan acciones señaladas por su familia o amigos, se acomodan a los problemas cuando se les presentan y ajustan su forma de ser si no va de acuerdo a la situación; estos mismos son los que realizan más acciones para conservar el medio ambiente. Este efecto apoya lo que se ha encontrado en otros estudios (Corral, 2010; Mayer et al., 2004; Palacios et al., 2011; 2012; Schmuck et al., 2003), al mencionar que los seres humanos somos parte de la naturaleza (Mayer et al., 2004), pero a la vez estamos generando un desequilibrio que pone en riesgo la propia supervivencia (Oskam, 2000; Schmuck et al., 2003). Sin embargo, se están realizando esfuerzos para evitar o minimizar los efectos de la acción humana en el mundo natural por medio de un conjunto de acciones que resulta en la protección del medio ambiente (Bustos et al., 2006; Corral, 2010; Palacios et al., 2012; Palacios et al., en prensa; Palacios et al., 2011).

$\mathrm{Al}$ analizar las semejanzas y diferencias con otros estudios, se puede decir que la gente reporta realizar más de una acción proambiental (Bustos et al., 2006; Bustos et al., 2008; Palacios et al., 2012, Palacios et al., en prensa), además de poner en marcha mecanismos adaptativos. Esto coincide con el trabajo de Griskevicius et al. (2012), quienes al realizar un análisis de literatura establecen la importancia de las variables evolutivas en la adaptación al cambio ambiental. Sin embargo, hay una diferencia: en nuestro estudio se mostró evidencia empírica de esa relación. Autores como Griskevicius et al. (2012) y Griskevicius et al. (2010) proponen como explicación que la adaptación a las nuevas condiciones ambientales, su protección y restauración, supone una combinación de tendencias adaptativas y estrategias psicosociales. Su propuesta coincide con las dimensiones encontradas en el esca- lamiento multidimensional del presente estudio y que ciertamente, en la actualidad se requieren propuestas de este tipo.

Los resultados obtenidos mediante la regresión por pasos sucesivos mostraron que la adaptación a nichos naturales y urbanos tiene un efecto significativo en las compras que realizan los jóvenes para ser más responsables con el medio ambiente. En otras palabras, quienes se ajustan a formar parte de la naturaleza, a convivir con ella y a los cambios de clima, así como los jóvenes que en su vida diaria se acostumbran al tráfico de la ciudad, la contaminación y se acostumbran a vivir con poca agua, son quienes adquieren productos más amigables con el ambiente y durables. Lo anterior encuentra sustento en lo que han reportado algunos autores (Brown, 2009; Bustos, 2004; Bustos et al., en prensa; Corral, 2010).

La aparición de los dos entornos (natural y urbano) como factores que predicen el consumo responsable, forman un continuo que va de lo urbano a lo natural y viceversa, tal y como se mostró en la configuración obtenida en el escalamiento, lo que parece indicar que aunque el entorno natural y urbano se oponen en el sentido de que el crecimiento del segundo implica la reducción del primero, los jóvenes están articulando ambos aspectos en el cuidado del medio ambiente al comprar ciertos productos que no lo afectan, lo que se traduce en acciones que tienen como finalidad el cuidado de los recursos naturales.

En este orden de ideas, la búsqueda de sustentabilidad se ha convertido en una estrategia clave para las generaciones actuales, las cuales tendrán a su vez el reto de enseñar a sus descendientes estilos de vida acordes con las exigencias de protección de los recursos escasos (Bustos, 2004; Bustos et al., en prensa). Adicionalmente, el hombre como ser natural, ha construido espacios que le permiten eliminar las amenazas del ambiente como una forma de lograr bienestar. El ajuste del medio urbano, por un lado, y del medio natural, por otro, son aparentemente nichos ecológicos indispensables para la adaptación de los jóvenes en términos de los resultados que aquí se han mostrado.

Al poner a prueba la hipótesis nula podemos afirmar que esta se rechaza, por lo tanto, es posible sostener que existe un efecto de las características de adaptación natural y sociocultural sobre acciones de con- 
servación ambiental, además de que la adaptación natural y urbana inciden en un consumo ambientalmente responsable por parte de los jóvenes de esta muestra. Un aspecto relevante de la hipótesis es que la conducta proambiental está significativamente asociada a la adaptación natural y sociocultural, lo que puede indicar que la CPA es vista como un grupo de comportamientos ligado al entorno natural y a la demanda por cuidarlo, así como a ajustar su forma de ser a la situación en la que se encuentran los jóvenes al encarar los problemas que se presentan cuando se produce un daño al medio ambiente. En tanto, la relación significativa entre la adaptación al entorno natural y al urbano con el consumo ambientalmente sustentable podría estar implicando que para cuidar la naturaleza hay que realizar un consumo sostenible, y esta oferta de consumo se genera principalmente en el medio urbano.

La muestra estudiada, sin el propósito de generalizar los resultados, evidenció una tendencia a combinar ajustes adaptativos a los nichos natural, sociocultural y urbano como factores que muestran asociación significativa con variables comportamentales de conservación y consumo responsable de recursos. Lo anterior le otorga validez predictiva a la escala de adaptación ambiental que se ha propuesto para este estudio y permite, en futuros estudios, obtener correlatos con la personalidad, la supervivencia, la elección de pareja y/o variables psicoambientales.

La adaptación ambiental incide en las acciones que realizan los jóvenes; esto podrá servir para implementar estrategias de intervención en donde se fomente la integración con el medio ambiental natural y la realización de conductas proambientales que ayuden a preservar el entorno tanto físico, como biológico y psicosocial. Las acciones del cuidado del medio ambiente, como formas de adaptación, podrán ser el centro de estrategias sociales y educativas con el fin de lograr sociedades más respetuosas del entorno natural y su conservación futura.

Al ser un estudio en etapa inicial, tiene algunas limitaciones que parece conveniente considerar. En primera instancia, la evidencia mostrada en el sustento teórico parece indicar la existencia de tres nichos ecológicos a los cuales las personas se pueden adaptar. Mediante el uso del escalamiento dimensional los resultados obtenidos mostraron que perceptualmente la solución más sencilla corresponde a dos dimensiones; sin embargo, los indicadores de bondad de ajuste señalaron que la solución más eficiente es la de cuatro dimensiones. De esta manera, los nichos ecológicos que se encuentran presentes en el ecosistema humano de los jóvenes mexicanos de esta muestra son el natural, el urbano, el sociocultural y el individual. Esta última dimensión parece mostrar conductas que aparecen en la vida cotidiana y social, que claramente corresponden al contexto sociocultural mexicano, como se reporta en otros estudios (Díaz-Guerrero, 1984, 1993, 1994; Díaz-Loving, 1998), y que permite estudiar conductas especificas en contextos culturales particulares como el nuestro (Palacios et al., en prensa; 2012; 2012, julio). En este sentido, el nivel de relación encontrado entre las estrategias adaptativas obtenidas a nivel individual y el sociocultural, describe las características de vinculación que los jóvenes utilizan en su entorno para poder ajustarse e interactuar con sus semejantes en estos entornos.

Con respecto a la forma de medición, si bien el escalamiento multidimensional se utiliza en algunas ocasiones para evaluar las preferencias o el grado de favorabilidad hacia algún producto; para la presente investigación ésta no fue la finalidad. A diferencia de la evaluación de preferencias, más bien, mediante la forma de medición utilizada se identificaron estrategias de adaptación que el hombre ha desarrollado para integrarse a ecosistemas en los que se encuentra.

Las variantes adaptativas aquí reportadas pueden verse como patrones de comportamiento de complejidad mayor que las acciones simples de conducta proambiental, como separar los residuos plásticos o consumir productos orgánicos. En este sentido, estaríamos hablando de haber encontrado asociaciones entre dos niveles de comportamiento. Otra explicación es que la variantes adaptativas obtenidas en el presente estudio son tendencias a la acción, más que acciones concretas, lo que permitiría suponer que la CPA es una consecuencia de dichas tendencias adaptativas, urbana, sociocultural y natural. Si esto es así, un elemento a considerar será replicar el estudio para corroborar lo obtenido, la realización de nuevos estudios se concibe como una meta importante y clarificadora.

Para la replica del estudio, sugerimos utilizar otras técnicas como el análisis de ecuaciones estructurales 
para corroborar las dimensiones obtenidas; además, proponemos aumentar la variedad y cantidad de afirmaciones con la finalidad de obtener un mayor nivel de consistencia interna. Aunado a lo anterior, futuros estudios permitirán mostrar la aplicación de los resultados para elaborar intervenciones psicoambientales sensibles y que persuadan a los jóvenes de conservar el medio ambiente.

Por último, hay que considerar la importancia que tiene la forma en que se relacionan las formas de adaptación encontradas en esta investigación con la conducta proambiental y el consumo ambientalmente responsable; y en ese sentido, lo encontrado permite generar las directrices sobre nuevos trabajos en esta línea. Es así que futuros estudios permitirán conocer si los mecanismos de adaptación se vinculan con la salud física y psicológica del individuo. Además, podremos conocer de qué forma el entorno social, los espacios

\section{REFERENCIAS}

Arce, C., Francisco, C. y Arce, I. (2010). Escalamiento multidimensional: concepto y aplicaciones. Papeles del Psicólogo, 31(1), 46-56.

Bechtel, R. B. (1997). Environment and behavior. An introduction. Thousand Oaks: Sage.

Brown, L. R. (2009). Plan B 4.0. Movilizing to save civilization. NY: Norton.

Buss, D. M. (1984). Evolutionary biology and personality psychology: Toward a conception of human nature and individual differences. American Psychologist, 39(10), 1135-1147.

Buss, D. M. (1991). Evolutionary personality psychology. Annual Review of Psychology, 42, 459-491.

Buss, D. M. (1995). Psychological sex differences: Origins through sexual selection. American Psychologist, 50, 164-168.

Buss, D. M. (1995). Evolutionary psychology: A new paradigm for psychological science. Psychological Inquiry, 6(1), 1-30.

Buss, D. M. (1996). Social adaptation and five major factors of personality. En J. S. Wiggins (Ed.). The fivefactor model of personality: Theorical perspectives (pp. 180-207). NY: Gilford. urbanos, la actividad cotidiana, comercial e industrial afectan al individuo, ya que colateralmente se tiene una incidencia en la contaminación del aire, agua y suelo, que implican un deterioro del ecosistema con amenazas para la salud y el bienestar de las personas.

Los resultados obtenidos hasta ahora permiten afirmar que los seres humanos somos adaptables a diferentes nichos y las características de personalidad ayudan a entender cómo el mundo diseñado por el hombre, e incluso el natural, tienen efectos sobre los comportamientos y las situaciones que se enfrentan. Debido a la existencia de estos efectos, se debe tomar en cuenta que el desequilibrio ambiental genera un deterioro de la calidad de vida de las personas, con efectos sobre la salud física, mental y social, de ahí la importancia de investigar cómo la adaptación del individuo promueve acciones de cuidado ambiental.

Buss, D. M. (2009). How can evolutionary psychology successfully explain personality and individual differences? Perspectives on Psychological Science, 4(4), 359- 366.

Buss, D. M. (2009). An evolutionary formulation of person-situation interactions. Journal of Research in Personality, 43(2), 241-242.

Buss, D. \& Barnes, M. (1986). Preferences in Human Mate Selection. Journal of Personality and Social Psychology, 50(3), 559-570.

Buss, D. \& Greiling, H. (1999). Adaptative individual differences. Journal of Personality, 62(2), 209-243.

Buss, D. \& Shackelford, T.K. (1997). Human aggression in evolutionary psychological perspective. Clinical Psychology Review, 17(6), 605-619.

Bustos, A. M. (2004). Modelo de conducta proambiental para la conservación de agua potable. (Tesis de doctorado no publicada). México: Universidad Nacional Autónoma de México.

Bustos, A. M. y Flores, H. L. M. (2006). Obligación de cuidar los recursos ambientales y actitud relacionadas con tres tipos de conducta proambiental. En Sánchez, A. R., Díaz-Loving, R. y Rivera, A. S. (Eds.). La Psicología Social en México (pp. 676- 683), vol. 11, México: Amepso. 
Bustos, A. M., Flores, H. L. M. y Barrientos, D. C. (2008). Estimación de la disposición ambiental. IV Congreso internacional de psicología social. Memoria en extenso. Psicología ambiental y desarrollo sustentable, pp. 1-11. Benemérita Universidad Autónoma de Puebla, 20-22 de noviembre.

Bustos, A. M., Palacios, D. J., Barrientos, D. C. y Flores, H. L. M. (en prensa).Validez de la escala de consumo ambientalmente responsable. El Psicólogo Anáhuac, 15.

Bustos, A. M., Ramos, H. F., Barrón, C. M., López, D. A., Ocaña, D. E. (2011, noviembre). Promoción de conducta proambiental en parques urbanos. Ponencia presentada en el Tercer Encuentro Latinoamericano de Psicología Ambiental, Tlalnepantla, México.

Corral, V. V. (2010). Psicología de la sustentabilidad. México: Trillas.

Corral, V. V., Tapia, C., Frías, M., Fraijo, B. \& González, D. (2009). Orientación a la sostenibilidad como base para el comportamiento pro-social y pro-ecológico. Medio Ambiente y Comportamiento Humano, 10(3), 195-215.

Corraliza, J. A. y Berenguer, J. (2010). Emoción y ambiente. En J. I. Aragonés, y M. Amérigo (2010). Psicología ambiental. Madrid: Pirámide.

Díaz-Guerrero, R. (1984). La psicología de los mexicanos: Un paradigma. Revista Mexicana de Psicología, 1(2), 95-104.

Díaz-Guerrero, R. (1988). Hacia una psicología ecosistémica. Revista de Psicología Social y Personalidad, 4(2), 65-81.

Díaz-Guerrero, R. (1993). Un factor cardinal en la personalidad de los mexicanos. Revista de Psicología Social y Personalidad, 9(2), 1-19.

Díaz-Guerrero, R. (1994). La psicología del mexicano (6a. Ed.). México: Trillas.

Díaz-Guerrero, R. (1995). Una aproximación científica a la etnopsicología. Revista Latinoamericana de Psicología, 27(3), 359-389.

Díaz-Guerrero, R. (2001). La teoría del ecosistema humano (3a. Ed.). En J. Cueli y L. Reidl (Eds.). Teorías de la personalidad (pp. 578-590). México: Trillas.

Díaz-Loving, R. (1998). Contributions of Mexican ethnopsychology to the resolution of the etic-emic dilemma in personality. Journal of Cross-Cultural Psychology, 29(1), 114-118.
Díaz-Loving, R. (2008). Etnopsicología mexicana. México: Trillas.

Díaz-Loving, R. (2011). Current Mesoamerican couples: Cultural heritage; families in transition; sustainable relationships. Acta de Investigación Psicológica, 1(2), 330-351.

Flores, X. R. y González-Guillén, M. J. (2007). Consideraciones sociales en el diseño y planificación de parques urbanos. Economía, Sociedad y Territorio, 6, 914-951.

Gifford, R. (2007). Environmental Psychology. (4a. Ed.), Optimal Books: Canadá.

Granada, H. (2003). La cultura como estrategia de adaptación en la interacción sujeto social-ambiente. Investigación y Desarrollo, 11(1), 134-161.

Griskevicius, V., Cantú, S. M. \& Van Vugt, M. (2012). The evolutionary bases for sustainable behavior: implications for marketing, policy, and social entrepreneurship. Journal of Public Policy \& Marketing, 31, 115-128.

Griskevicius, V., Shiota, M. \& Nowlis, S. (2010). The many shades of rose-colored glasses: An Evolutionary approach to the influence of different positive emotions. Journal of Consumer Research, 37. doi: 10.1086/ 651442

Griskevicius, V., Tybur, J. \& Van den Bergh, B. (2010). Going green to be seen: Status, reputation, and conspicuous conservation. Journal of Personality and Social Psychology, 98(3), 392-404.

Hair, J., Anderson, R., Tatham, R. y Black, W. (1999). Análisis Multivariante. (5a. Ed.). Madrid: Prentice Hall.

Hartig, T. (2011). Issues in restorative environments research: matters of measurement. En B. FernándezRamírez, V. C. Hidalgo, F. C. Salvador y M. J. Martos (Eds.) Psicología ambiental 2011: entre los estudios urbanos y el análisis de la sostenibilidad. Almería: Universidad de Almería.

Home, R., Bauer, N. \& Hunziker, M. (2010). Cultural and biological determinants in the evaluation of urban green spaces. Environment and Behavior, 42(4), 494523.

Landázuri, A. M., Lee, T. R., Terán, A. \& Mercado, D. S. (2010). Green areas and housing's habitability. En M. Bonaiuto, M. Bonnes, A. M. Nenci \& G. Carrus (Eds.). Advances in people-environment studies. Vol. 2 (pp. 125-135). Götingen, Germany: Hogrefe. 
Lazarus, R. y Folkman, S. (1986). Estrés y procesos cognitivos (pp. 164-187). España: Martínez Roca.

Li, N., Griskevicius, V., Durante, K., Jonason, P., Pasiszand, D. \& Aumer, K. (2009). An evolutionary perspective on humor: Sexual selection or interest indication? Personality and Social Psychology Bulletin, 35(7), 923-936. doi: 10.1177/0146167209334786

MacDonald, K. (1995). Evolution, the Five-Factor Model, and levels of personality. Journal of Personality, 63(3), 525-567.

Martín, R. E. (1993). Adaptación y adaptabilidad de las poblaciones prehistóricas canarias. Una primera aproximación. Vegueta, 1, 9-19.

Martínez, J. y Montero, M. (2010). Percepción de cualidades restauradoras y preferencia ambiental. Revista Mexicana de Psicología, 27, 183-190.

Mayer, S. \& Frantz, C. (2004). The connectedness to nature scale: A measure of individuals' feeling in community with nature. Journal of Environmental Psychology, 24, 503-515.

Mercado, D. S, Landázuri, A. M. y Terán, A. (2006). Psicología ambiental, una visión en perspectiva. En L. M. Flores y M. Bustos (Eds.). Concepciones de la interacción social en el niño (pp. 23-77). México D. F.: FES Zaragoza, UNAM.

Oskam, S. (2000). A sustainable future for humanity? American Psychologist, 55(5), 496-508.

Palacios, D. J. y Bustos, A. J. M. (en prensa). Validez factorial de la autoeficacia ambiental y su influencia estructural sobre la conducta proambiental en jóvenes. Revista Iberoamericana de Evaluación en Psicología.

Palacios, D. J. y Bustos, A. J. M. (2011). Análisis de la autoeficacia ambiental en el manejo de residuos sóli- dos, comparación en hombres y mujeres. Revista El Psicólogo Anáhuac, 14, 31-38.

Palacios, D. J. y Bustos, A. J. M. (2012). Modelo de autoeficacia y habilidades ambientales como predictores de la intención y disposición proambiental en jóvenes. Revista Intercontinental de Psicología y Educación, 14(2), 143-163.

Palacios, D. J. y Bustos, A. J. M. (2012, julio). Aproximaciones de interdependencia con el ambiente: $\mathrm{Su}$ inclusión en el yo y la cercanía con la naturaleza. Ponencia presentada en el V Coloquio de Experiencias de Investigación en Psicología Ambiental. México: fes Zaragoza, unAM.

Schmitt, D. (2008). Evolutionary perspectives on romantic attachment and culture: How ecological stressors influence dismissing orientations across genders and geographies. Cross-Cultural Research, 42, 3, 220-247.

Schmuck, P. \& Vlek, C. (2003). Psychologist can do much to support sustainable development. European Psychologist, 8(2), 66-76.

Tal, I., Hill, D., Figueredo, A. J., Frías, A. M. \& Corral, V. V. (2006). An evolutionary approach to explaining water conservation behavior. Medio Ambiente y Comportamiento Humano, 7(1), 7-27.

Tobby, J. \& Cosmides, L. (1990). On the universality of human nature and the uniqueness of the Individual: The Role of Genetics and Adaptation. Journal of Personality, 58(1), 1767.

Triandis, H. C. (1996). The psychological measurement of cultural syndromes. American Psychologist, 51(4), 407-415. 